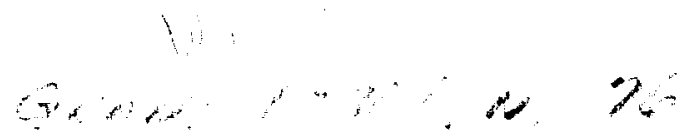

\title{
Cuntrol of Melt Convection Using Traveling Magnetic Fields
}

\author{
Konstintin Mizzurnk \\ VASA Marmhall Spare Flight Center \\ Hunterilk. AL 3580:3, USA
}

Abstract

An axismuntriv traveing magnetic wave induces a meridional base flow in a cylindrical zone of an electrically conducting liquid. This remotely induced flow can be conveniently controlled, in magnitucle and direction. and can have benefits for crystal growth applications. In particular. it call be nised to siguificantly offset natural convection. Theoretical basics of this new technological merhod are presented.

\section{Introduction}

Buojancy conroction, usually present during single crystal growth, is known to play a crucial role in defect formation and quality of the crystal. It originates from externally imposed thermal and/or solutal gradients necessary for crystallization, and the presence of gravity. Considerable research has been focused on controlling convection with the aim of improving macro- and microscopic homogeneity of the grown material. Utilizing a microgravity environment is the obvious solution to significantly reduce the gravity level and hence convection. Another method involves the application of static or dynamic magnetic fields. This method can be used only for electrically conducting melts, such as liquid metals and semiconductors, as well as ionic solutions or melts. While static magnetic fields can only play a passive role to damp already existing flows, time dependent magnetic fields can set up a steady state Lorentz body force, inducing flow in the system. The most recognized example is the rotating magnetic field (RMF). Over the past decade, RMF has been extensively studied as means for generating convective patterns beneficial to solidification processing $[1,2]$. It has been used for such growth techniques as vertical Bridgman(VB), float zone(FZ), or the traveling heater method (THM). The molten zone in these techniques employs a cylindrical geometry. When RMF is applied to such a zone, it induces a swirling, rotating, flow. This flow, in turn, builds up a secondary flow in a form of two rolls, one adjacent to the bottom of the molten carity, and the other at the top. The secondary flow direction is always pointing into the melt at the central axis. The benefits of this type of flow pattern are: a) the azimuthal flow homogenizes and reinforces axisymmetry in the melt, b) it may suppress oscillatory buoyancy convection. Crystals grown in presence of RMF are reportedly of better quality [3-5]. We have recently proposed yet another type of magnetic field configuration that may find use in crystal growth practices[6,7]. It consists of an axisymmetric traveling magnetic wave that induces a steady state Lorentz force in the axial direction. When applied to a long open tube, it will pump the fluid. For closed cavities, a recirculating meridional flow is set up. These are essential features of the traveling magnetic field (TMF) method. Consider now a VB configuration with moderate radial thermal gradients (Grashof number $\mathrm{Gr}$ of the order of $10^{5}$ or less). Natural convection will then consist of a single axisymmetric roll. Application of TMF to this system will significantly modify the flow pattern. 


\begin{abstract}
क...

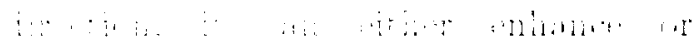

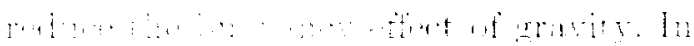

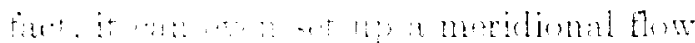

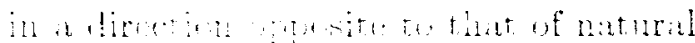
anvertion. The posibility may be attration for in atiue shape control. relucing concarity. for eximple. A practival implementation of the technicue consists of a stack of windings around the cylinder. A controlled phase shift of the applied electric power to each of these segments can result in a propagating magnetic wave. The power incel, the frequency, the spacing between the sections and direction of propagation are the control parameters of this technique. Further feasible modifications include axially non-uniform TMF, TMF in combination with the static magnetic fields, TMF plus RMF, etc.
\end{abstract}

Possible materials processing applications of TMF include $\mathrm{VB}, \mathrm{FZ}$ and THM crystal growth methods. TMF can be useful also in homogenizing melts in long cylindrical ampoules prior to solidification, an especially useful feature when processing in a microgravity environment. Finally, it can be used to control the sedimentation process taking place in ionic solutions.

In this work we examine the possibility of counter-balancing natural convection by application of TMF for VB and THM configurations. In order to demonstrate damping of the natural convection effect, we consider here a case of VB with germanium in a silica ampoule. We numerically obtain the buoyancy force distribution and then consider a TMF induced force superimposed upon it. The combined force can be, for example, optimized is such a way that the flow in the vicinity of the growing interface is minimized. Also, a model of THM with applied T.MF is presented and a buoyancy convective flow reduction as much as 50 times is demonstrated.

\section{Theoretical braticas}

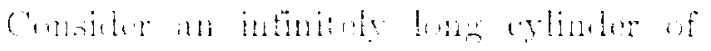

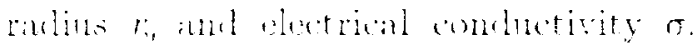
We further assume that the surromeling cylinder medium is an insulator and a non-magnetic material. An external axisymmetric magnetic wave is applied to this cylinder along its axis. The frequency of this field is such that no proparating electromagnetic field is generater in the surrounding cylinder space. In this particular case, the magnetic field can be conveniently represented by its vector potential $\mathbf{A}$, as it has only an azimuthal component. A single harmonic wave propagating in the $z$-direction along the axis of the cylinder of the frequency $\omega$ and the wave-vector $a$ can be represented as:

$$
\mathbf{A}=\mathbf{e}_{\psi} A(r) e^{\mathbf{x}(\omega t-a z)}
$$

Here we follow the notation that the real parts of the complex variables correspond to physical quantities. The following well-known set of electromagnetic equations is employed in our model:

$$
\begin{aligned}
& \mathbf{B}=\nabla \times \mathbf{A}, \quad \nabla \times \mathbf{H}=\mathbf{j} \\
& \nabla \times \mathbf{E}=-\frac{\partial \mathbf{B}}{\partial t}, \\
& \mathbf{E}=-\nabla \phi-\frac{\partial \mathbf{A}}{\partial t}
\end{aligned}
$$

where $\mathbf{B}, \mathbf{E}$ and $\phi$ are the magnetic inductance, electric field, and electric potential, respectively. It is convenient to select the Coulomb gauge for $\mathbf{A}$ : $\nabla \mathbf{A}=0$. The electric current density induced in the electrical conductor can be written in the following general form:

$$
\mathbf{j}=\sigma\left(-\nabla \phi-\frac{\partial \mathbf{A}}{\partial t}+\mathbf{v} \times \mathbf{B}\right)
$$

where $\mathbf{v}$ is the local velocity of the 


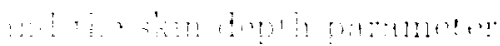

$\therefore \quad r \quad y+1$

$51-\operatorname{tat}\left|\nabla+\frac{j 1}{j t}\right|$

and using the selected gauge for $\mathbf{A}$ we gnt for the dectric potential $\nabla^{2} \delta=0$.

As both $\mathbf{j}$ and $\mathbf{A}$ have only the azimuthal components, then components of these vectors normal to the interface are antomatically zero. Consequently. the derivative of $o$ normal to the interface is also zero, and in such a case the solution for $\phi$ is const, which can be set to zero. Substituting a general axisymmetric form (1) into Eq. (4) results in the following differential equation:

$\left(\frac{\partial^{2}}{\partial r^{2}}+\frac{1}{r} \frac{\partial}{\partial r}-\frac{1}{r^{2}}-a^{2}-i \omega \sigma \mu\right) A=0$

with the boundary condition of continuity for $A$ together with its normal derivatives across the interface.

It is convenient to introduce a nondimensional scaling of the distance. The most natural scale in our model is the cylinder radius $r_{0}$ and it is adopted for the rest of the paper. The resultant differential equation (5) can be easily integrated and leads to the following solution:

$$
\begin{aligned}
& A_{\text {iuf }}(r)=\left\{\begin{array}{c}
A_{\text {in }} I_{1}(\gamma r) \\
A_{0} I_{1}(a r)+\left\lfloor A_{\text {in }} \frac{I_{1}(\gamma)}{K_{1}(a)}-A_{0} \frac{I_{1}(a)}{K_{1}(a)}\right] K_{1}(a r)
\end{array}\right. \\
& \text {, } r<1 \\
& \text {, } r>1
\end{aligned}
$$

where

$$
\begin{aligned}
& A_{i n}=\frac{A_{0}}{\gamma I_{0}(a) K_{1}(a)+a I_{1}(a) K_{0}(a)}, \\
& \gamma=\sqrt{a^{2}+i K^{2}},
\end{aligned}
$$

This finde induces an not Lorentz force in the stationary modium with the steadystate component in the z-direction:

$$
\left\langle F_{z}\right\rangle=\frac{\omega \sigma a}{2}\left|A_{: n}\right|^{2}\left|I_{1}(\gamma r)\right|^{2}
$$

For the low frequency regime, $K<<1$, we obtain $\left\langle F_{r}\right\rangle=0$ and

$$
\left\langle F_{z}\right\rangle=\frac{\omega \sigma a}{2} A_{\mathrm{v}}^{2} I_{1}^{2}(a r)
$$

Note that this low frequency limit formula is valid for a finite cylinder geometry as well. In this limit, the induced magnetic field is neglected. For an infinite geometry, as frequency increases, the $r$-component of the force becomes noticeable and squeezes the cylinder. For a finite cylinder geometry, the numerical solution of the induced Lorentz force indicates significant cap effects for values of $K$ in the range of $3-$ 10 [9]. In this work, we confine analysis to low frequencies, $K<1$, which is often the case for practical purposes.

\section{Numerical work}

The TMF concept emerged from recognizing deficiencies in the RMF technique to generate an optimal flow for crystal growth in cylindrical ampoules. RMF can be viewed as a magnetic wave propagating in the azimuthal direction along a closed path. The obvious requirement here is that admissible values for the wave-vector are integers; the other geometries involve open paths. The waves can be planar, axisymmetric, or more complex. 


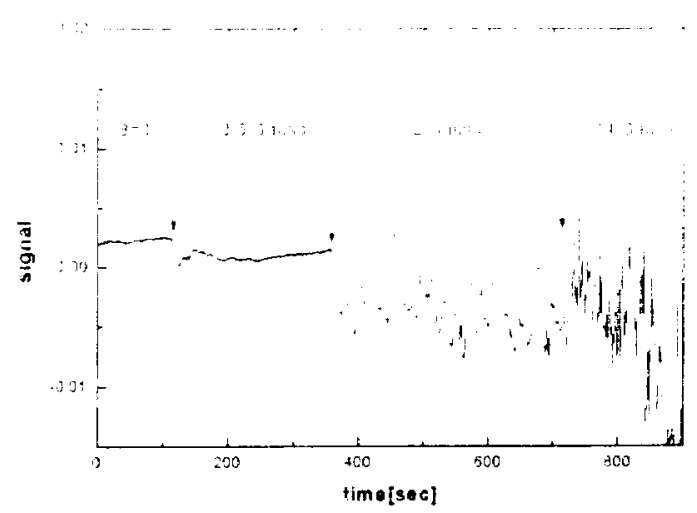

Fig.1. The anemometer response to a duwnward traveling magnetic field for three different values of applied magnetic field.

However, the axisymmetric case is particularly attractive as it can be easily obtained and has potential applications.

In order to demonstrate the basic principles of this novel technique, we conducted an experiment with a cylindrical column of mercury[6]. The radius of the column is $3.175 \mathrm{~cm}$ and its height is $12 \mathrm{~cm}$. A stack of six coils was wound around the column with a $2.5 \mathrm{~cm}$ length of a single section. The frequency of the applied voltage was $50 \mathrm{~Hz}$. The flow response to the TMF strength was studied by utilizing a hot film anemometer. Fig.1 represents the anemometer signal evolution as the applied TMF power increases stepwise from zero. The rms value of the applied magnetic field was measured at the surface of the column. A clear transition from the steady to oscillatory flow occurs between 3.5 and 7.2 Gauss. Incidentally, this value is close to the unsteady flow transition for the RMF technique. Application of TMF power above 14 Gauss results in turbulent motion. A millitesla level of the magnetic field required to induce a significant flow is an advantage of this technique. The induced Lorentz force is in the range of the buoyancy force

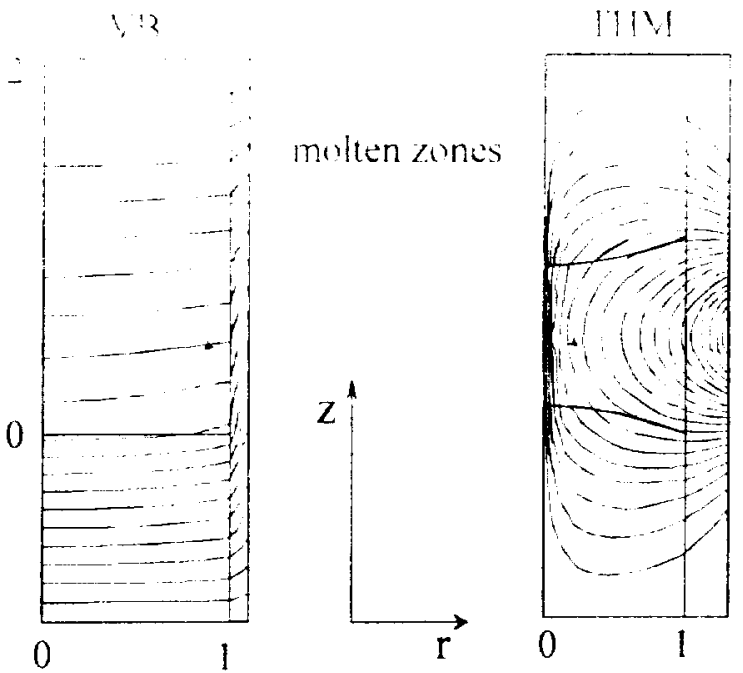

Fig. 2. The geometry and thermal field contours of the VB and THM cells used for numerical modeling.

commonly encountered in solidification processing. It becomes then apparent that the TMF can potentially counterbalance the buoyancy force. A stagnant layer can be formed at the growing interface, or the flow direction can be reversed. Clearly, accurate balancing the two forces can be experimentally challenging: a flow transducer may be required for this purpose. Flow reversal can be accomplished with less difficulty. Experimental work in this direction has just begun, and our goal here is to numerically demonstrate the feasibility of damping convection by TMF. VB and THM models are selected for this mostly illustrative purpose. The analysis is further restricted to the steady-state axisymmetric case. Also, the low frequency approximation (9) is adopted for this calculation. The Lorentz force averaged over one oscillation for the non-zero motion of the medium contains terms linear in velocity which are not present in Eq.(9). With these terms included, the following equation for the vorticity, induced by a combination of 


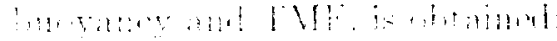

$$
\begin{aligned}
& \frac{\partial m}{\partial t}=\left[\frac{r^{2}}{\partial r^{\prime}}+\frac{1}{r} \frac{\partial}{\partial r}-\frac{1}{r^{2}}+\frac{\partial}{\partial \partial^{2}}\right] w+\frac{v_{1} u}{r} \\
& -\left(v \frac{\partial}{\partial z}+v_{r} \frac{\partial}{\partial r}\right) w-H a^{2} I_{i j}^{2}(a r) \frac{\partial v_{1}}{\partial z} \\
& +\frac{\partial}{\partial r}\left(\left(H a^{2} v_{-}-\frac{T m}{a}\right) I_{1}^{2}(a r)-G r \theta\right) \\
& \operatorname{Pr}\left(\frac{\partial \theta}{\partial t}+v_{r} \frac{\partial \theta}{\partial r}+v_{z} \frac{\partial \theta}{\partial z}\right)=\nabla^{2} \theta
\end{aligned}
$$

where the adopted here usual notation and scaling is:

$$
\begin{aligned}
& v=v^{\prime} \nu / r_{0}, \quad w=w^{\prime} \nu / r_{0}^{2}, \quad t=t^{\prime} r_{0}^{2} / \nu, \\
& \theta=\frac{T-T_{b o t t}}{T_{\text {top }}-T_{\text {bott }}}, \quad G r=\frac{g \beta r_{0}^{3}}{\nu^{2}}\left(T_{t o p}-T_{b o t t}\right), \\
& T m=\frac{\sigma \omega a^{2} A_{0}^{2} r_{0}^{4}}{2 \rho \nu^{2}}, \quad H a^{2}=\frac{a^{2} A_{0}^{2} \sigma r_{0}^{2}}{2 \rho \nu}, \\
& \operatorname{Pr}=\frac{\nu}{\kappa} .
\end{aligned}
$$

where $T_{\text {top }}$ is the temperature at the top of the cell, $T_{\text {ivtt }}$ is the temperature at the bottom of the VB cell or at the center of the side wall for the THM cell, $P r$ is the Prandtl number, $T m$ is the magnetic an:

The wentel ablation (100) represents the hat transport equation needed for bnomalle concertion andysis. Additional. not explicitly displayed. equations are the incompressibility condition and the definition of the vorticity. In general, we have a multiparametric problem described by a set of non-dimensional parameters: $\operatorname{Pr} . \mathrm{Gr}$. $T m, H a, L, a, K$. We confine our analysis here to the case of $P r=0.1, a=1$. $K<1, H a-1$, and the aspect ratio $L=1$. Fig. 2 represents the geometry and thermal profiles for both the VB and THM models. The VB model describes molten germanium above a germanium crystal enclosed in a quartz ampoule. The temperature along the external wall of the ampoule is assumed to vary linearly and the top and bottom surfaces are isotherms. The ratio of thermal conductivities of the three materials, molten Ge, solid Ge, and silica is $51: 16.7: 1.5$, correspondingly. Due to this significant difference in thermal conductivities, the radial thermal gradient sets up at the growing crystal interface and induces natural convection. For Grashof numbers less
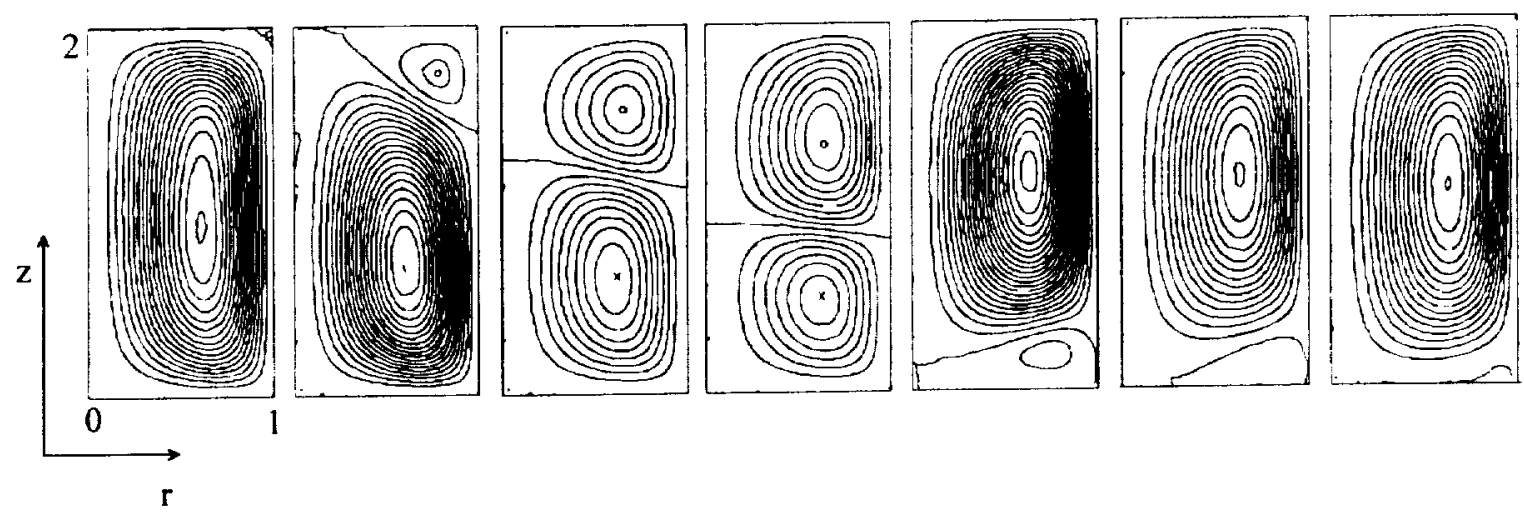

Fig. 3. A stream function contour plot of meridional flow induced in the VB cell by a combination of buoyancy and TMF force. The TMF applied force increases from the left to the right. At the $5^{\text {th }}$ and $6^{\text {th }}$ plots a stagnant fluid layer is formed at the bottom of the cell. 


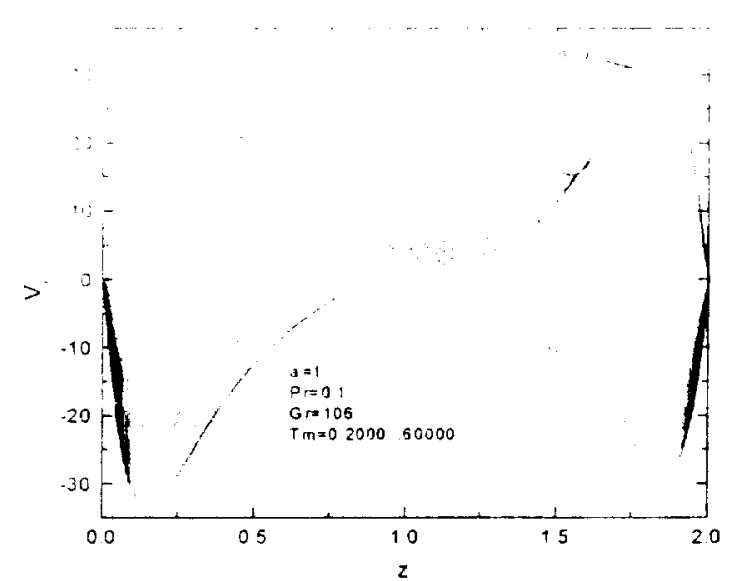

Fig.4. Profiles of the r-component of flow along the $z$ axis at $r=0.6$ for $V B$ configuration and for 30 values of $\mathrm{Tm}$.

than $10^{6}$. convection is steady and laminar[8]. The driving convection term in the vorticity equation (10a) is significantly non-uniform in the $z$ direction and it is confined to the growth interface area. In order to effectively counter-balance this term, the TMF term also has to be non-uniform. In this work a simplified case of the TMF, uniform in the z-direction. is considered. A commercial finite element with cubic basis numerical solver has been utilized for this work and adaptive gridding technique with approximately 10000 nodes is implemented.

When power of the TMF is applied in a direction opposite to the gravity force, it creates a small vortex at the top of the molten zone. At the same time, the flow in the buoyancy-induced vortex diminishes. As the TMF strength increases, the top vortex expands, until a stagnant layer is formed at the bottom. Further increase of the TMF field will set up a single vortex with the flow in a reverse direction to that of the initial natural convective flow. A two times reduction of the maximum flow velocity in the cell is obtained for some intermediate value of $T \mathrm{~m}$. The stream function evolution of this process is

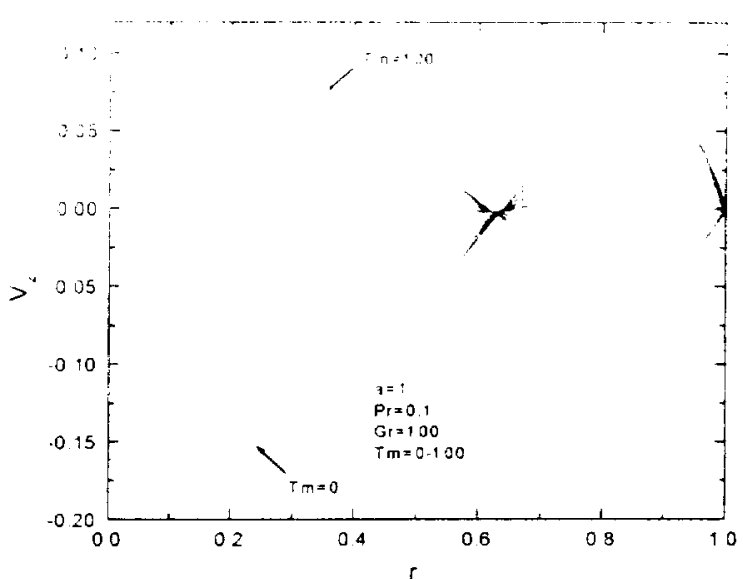

Fig.5. Profiles of the z-component of flow along the radius of the THM cell at $\mathrm{z}=0$ for a series of $\mathrm{Tm}$ values.

depicted in Fig. 3 and the $r$-component of the flow as a function of $z$ for $r=0.6$ and for a set of $T m$ values is presented in Fig.4. Clearly, a stagnant layer at the growth interface is observed. However, only for a narrow range of the magnetic Taylor numbers can its presence be detected.

Our THM model consists of a central molten zone between the two solids encased into the cylindrical ampoule as depicted in Fig.2. For the sake of simplicity, all three thermal conductivities are taken equal. At the side wall, a gaussian thermal profile is imposed and both the top and bottom surfaces are isotherms (see Fig. 2). The radial thermal gradient sets up a natural convection in the cell in a form of a single roll. As the vertical dimension of the molten zone is significantly lower than in the VB case, counter-balance of the buoyancy force by the uniformity in $z$-direction TMF can be more successful. Indeed, a significant reduction of the global flow is obtained. Fig. 5 represents a set of profiles of the $z$-component of flow velocity along the horizontal central line $(z=0, r)$ for an equidistant sequence of $T m$ values. A damping of original flow as much as 50 times is obtained. 


\section{Summmorms}

In this werk we hate numerieally demonstrater the feasibility of significantly riffectting gravity induced Hows by the application of axisymmetric traveling mangetic fields. For melts with small aspect ratios, the global flow can be ciramatically reduced, as demonstrated here. by 50-fold with THM application. For long cylinders convective flows induced by localized bouyancy forces are not effectively reduced by application of uniform TMF. However. a stagnant layer of fluid adjacent to the crystal growth interface can be obtained. A better approach is to consider a non-uniform localized TMF. In fact, only four coils are sufficient to set up a traveling magnetic field. Finally we want to note that in many instances, a steady state single axisymmetric vortex flow is beneficial during solidification processing. In such cases TMF can be a solution.

\section{Acknowledgements}

This work was done under the auspices of the Alliance for Microgravity Materials Science and Applications through NASA cooperative agreement NCC8-66. The author is grateful to Dr. Richard Grugel for his critical reading, fruitful discussions, and taking interest in this subject.

\section{REFERENCES}

1. P.Dold, K.W.Benz, "Rotating magnetic fields: fluid flow and crystal growth applications", Progress in Crystal Growth and Characterization of Materials 38(1999) 7-38
$\therefore \quad \because, \cdots, \cdots, \cdots, \cdots, \cdots, \eta_{1}, \cdots$,

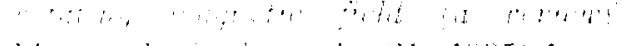 1.

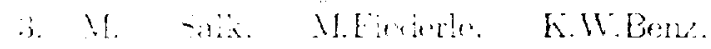

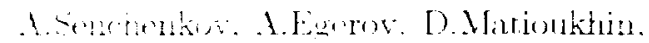

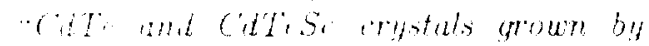 the irorchleng houtor metiod using a rotuting magneter ficld'. J. Crystal Growth 138 (1994) 161.

4. P. Dold. K.IV. Berz. ". Modification of fluid fou and heat transport in vertical Bridyman configurations by rotating magnete ficlds". Cryst. Res. Technol. $32(1997) 31$.

5. A.Senchenkov, I.Barmin. A.Tomson, V.Krapukhin, "Seedless THM growth of $\quad \mathrm{Cd}_{x} \mathrm{Hg}_{1 . x} \mathrm{Te}(x=0.2)$ single crystals within rotating magnctic ficld", $\mathrm{J}$. Crystal Growth 197 (1999) 552.

6. K. Mazuruk, N. Ramachandran, M.P. Volz. "Traveling Magmetic Field Induced Flou for Crystal Growth Applications", Proceedings of the $37^{\text {th }}$ AIAA Aerospace Sciences Meeting, Reno, NV, AIAA paper 99-0941, (1999).

7. X.Ramachandran, K.Mazuruk, M.P.Volz, "Use of traveling magnetic fields to control melt convection", J.Jpn.Soc.Microgravity Appl. 17 (2000) 98-103.

8. Ph.Marty, M.Witkowski. P.Trombetta, T.Tomasino, "On the stability of rotating MHD flows", Transfer Phenomena in Magnetohydrodynamic and Electroconducting Flows, A.Alemany et al.(eds.), 1999 Kluwer Academic Publishers, 327-343.

9. K.Mrzuruk, "Lorentz body force induced by traveling magnetic fields", Magnetohydrodynamics, subm. 